\begin{tabular}{|r|l|}
\hline \multicolumn{2}{|c|}{ Statistica Sinica Preprint No: SS-2020-0310 } \\
\hline Title & Inferring Social Influence in Dynamic Networks \\
\hline Manuscript ID & SS-2020-0310 \\
\hline URL & http://www.stat.sinica.edu.tw/statistica/ \\
\hline DOI & $10.5705 /$ ss.202020.0310 \\
\hline Complete List of Authors & $\begin{array}{l}\text { Xiang Cui and } \\
\text { Yuguo Chen }\end{array}$ \\
\hline Corresponding Author & Yuguo Chen \\
\hline E-mail & yuguo@illinois.edu \\
\hline Notice: Accepted version subject to English editing. \\
\hline
\end{tabular}


Statistica Sinica

\title{
INFERRING SOCIAL INFLUENCE IN DYNAMIC NETWORKS
}

\author{
Xiang Cui and Yuguo Chen \\ University of Illinois at Urbana-Champaign
}

\begin{abstract}
One interesting problem in social network analysis is whether individuals' behaviors or opinions can spread from one to another, which is known as social influence. The degrees of influence describes how far the influence can pass through individuals. In this paper, we explore the degrees of influence in dynamic networks. We build a longitudinal influence model to specify how people's behaviors can be influenced by others in a dynamic network. In order to determine the degrees of influence, we propose a sequential hypothesis testing procedure and use generalized estimating equations to account for multiple observations of the same individual across different time points. In addition, we show that the power of our proposed test goes to one as the network size goes to infinity. We illustrate the performance of our proposed method in simulation studies and real data analysis.

Key words and phrases: Degrees of influence, Dynamic network, Generalized estimating equations, Longitudinal analysis, Social influence.
\end{abstract}




\section{Introduction}

Social network analysis has become popular in many fields, including sociology, psychology, computer science and statistics. A social network consists of individuals and the relationships between them, which can be represented by nodes and edges in a graph, respectively. Social networks can be static or dynamic. A static network is a snapshot of a network at a certain time point, and a dynamic network is a sequence of observations of networks at different time points.

One interesting problem in social network analysis is whether the behaviors or opinions of an individual can be influenced by others in the network, which is known as social influence or social contagion. Some methods have been developed to study the spread of individuals' behavior within a social network (Valente, 1995; Centola, 2010). In addition, researchers have examined the spread of various individual health outcomes including obesity (Christakis and Fowler, 2007), smoking (Christakis and Fowler, 2008), sleep loss and drug use (Mednick, Christakis and Fowler, 2010), alcohol consumption (Rosenquist et al., 2010) and sexual orientation (Brakefield et al. 2014). See Sun and Tang (2011) for a summary of the models and algorithms developed for social influence analysis. Kempe, Kleinberg and Tardos (2003) proposed methods for selecting the most influential nodes in 
a network to maximize the spread of the influence (i.e., the social influence maximization). O’Malley (2013) used instrumental variables to account for the confounding effect in analyzing peer effects, and proposed a network influence model with multiple types of relationships.

The degrees of influence (DOI) describes how far the influence can pass through individuals in a network. For static networks, Christakis and Fowler (2013) proposed a permutation test to identify the behavior association between individuals across a social network using the Framingham Heart Study data, and they claimed that the spread of influence in social networks obeys the Three Degrees of Influence Rule. VanderWeele (2013) discussed three distinct interpretations of the Three Degrees of Influence Rule. However, O’Malley (2013) pointed out some issue with the choice of the null hypothesis in Christakis and Fowler (2013), and later $\mathrm{Su}$ (2019) proposed a new sequential test procedure with more appropriate null hypotheses to determine the degrees of influence.

The existing work can only detect the degrees of influence for static networks (Christakis and Fowler, 2013, $\mathrm{Su}, 2019$, and it is not clear how to extend their methods to dynamic networks. In this paper, we introduce a longitudinal influence model and a sequential hypothesis testing procedure to determine the degrees of influence for dynamic networks. We also provide 
theoretical properties regarding the level and power of the proposed test. In particular, we show that the power of the proposed test goes to one as the network size goes to infinity.

The remainder of the paper is organized as follows. Section 2 provides the basic notations. Section 3 introduces the longitudinal influence model. Section 4 gives the proposed sequential hypothesis testing procedure. Section 5 provides theoretical properties of the proposed method. Section 6 describes the simulation studies. Section 7 reports the results for the Higgs Twitter dataset (De Domenico et al. 2013) and the Digg dataset (Hogg and Lerman, 2012). Section 8 concludes the paper with a discussion.

\section{Notation}

Consider a dynamic social network consisting of $n$ individuals (nodes) and a set of dyadic relationships (edges) between them at time $t=1,2, \ldots, T$. We are mainly concerned with directed networks with no loops (both ends of an edge connect to a single node) or multiple edges between a pair of nodes. Such a dynamic network can be represented by its adjacency matrix $A_{t}=\left(a_{i j, t}\right)_{n \times n}, t=1,2, \ldots, T$, where each $A_{t}$ is an $n \times n$ binary square matrix with $a_{i j, t}=1$ if there is a directed edge from node $i$ to $j$ at time $t$ (i.e., individual $i$ is following individual $j$ at time $t$ ), and $a_{i j, t}=0$ otherwise. 
For a directed network, $A_{t}$ does not need to be symmetric.

In addition, we also observe whether each individual in the network possesses a specific trait, such as obesity, smoking, and happiness, at each time point $t$. This can be modeled by a binary random vector $Y_{i}=$ $\left(y_{i, 1}, y_{i, 2}, \ldots, y_{i, T}\right)$, with $y_{i, t}=1$ indicating the trait is present in individual $i$ at time $t$ and 0 otherwise. A toy example of such dynamic network is given in Figure 1 .

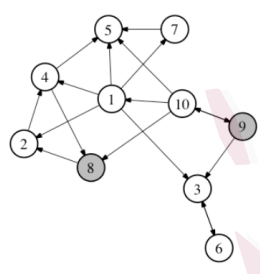

$t=1$

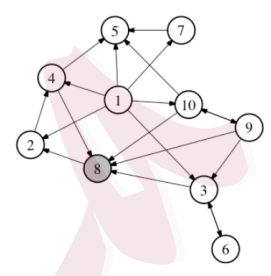

$\mathbf{t}=\mathbf{2}$

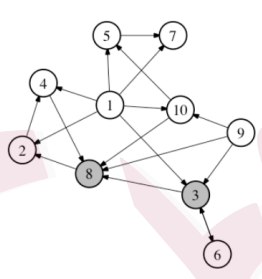

$\mathbf{t}=\mathbf{3}$

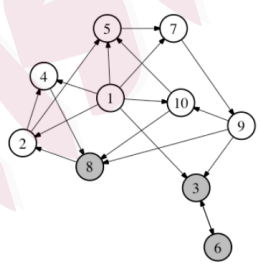

$\mathbf{t}=\mathbf{4}$

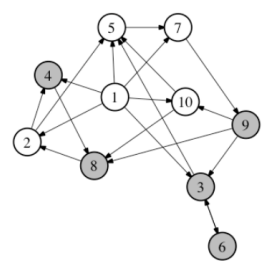

$\mathbf{t}=\mathbf{5}$

Figure 1: A toy example of a dynamic social network with gray nodes denoting smokers $\left(y_{i, t}=1\right)$ and white nodes denoting non-smokers $\left(y_{i, t}=\right.$ $0)$. 
In social networks, the individual we are focusing on is called the ego. If there is a directed path from the ego to an individual at time $t$, then that individual is called an alter. If the shortest directed path from the ego to an alter is $d$ at time $t$, then this alter is referred to as a $d$ th-degree alter, denoted by alter $_{d, t}$. Here is a simple illustration:

$$
\text { ego } \rightarrow \text { alter }_{1, t} \rightarrow \text { alter }_{2, t} \rightarrow \cdots
$$

Obviously the first-degree alters $\left(\right.$ alter $\left._{1, t}\right)$ are directly connected to the ego. The second-degree alters $\left(\right.$ alter $\left._{2, t}\right)$ have a length-2 path from the ego but they are not directly connected to the ego. In other words, $a_{\text {ego, } \text { alter }_{1, t}, t}=1$,

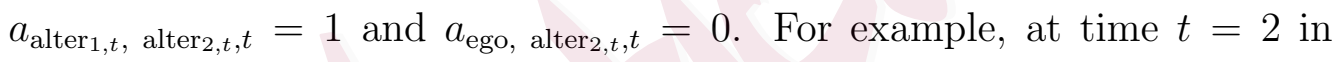
Figure 1, individuals 6 and 8 are first-degree alters of ego 3, individual 2 is a second-degree alter of ego 3 , and individual 4 is a third-degree alter of ego 3 , etc.

Let $d_{i j, t}$ be the length of the shortest directed path from $i$ to $j$ at time $t$, then $d_{\text {ego, } \text { alter }_{1, t}, t}=1, d_{\text {ego, } \text { alter }_{2, t}, t}=2$, etc. We define the $d$ th-degree alter set for each ego $i$ at time $t$ as:

$$
S_{i, t}^{d}=\left\{j: d_{i j, t}=d\right\}
$$

For an individual $i$ at time $t$, we define the $d$ th-degree influence factor $x_{i, t}^{d}$ 
as the average status of individual $i$ 's $d$ th-degree alters at time $t$, i.e.,

$$
x_{i, t}^{d}= \begin{cases}\frac{1}{\left|S_{i, t}^{d}\right|} \sum_{j \in S_{i, t}^{d}} y_{j, t}, & \left|S_{i, t}^{d}\right|>0 \\ 0, & \left|S_{i, t}^{d}\right|=0 .\end{cases}
$$

\section{Longitudinal Influence Model}

Social influence describes the process by which an individual's behavior or opinion is affected by others in the network. The influence may go beyond the people a person is directly linked to. We are interested in studying the degrees of influence, which describes how far the influence can pass through links between individuals. In this section, we specify a longitudinal influence model for different degrees of influence in dynamic networks.

We assume that

$$
y_{i, t} \sim \operatorname{Bernoulli}\left(p_{i, t}\right), \quad i=1,2, \ldots, n \text { and } t=1,2, \ldots, T \text {. }
$$

If the degrees of influence is 0 , then the behavior of each individual is not affected by others in the network. Therefore $y_{i, t+1}$ only depends on individual $i$ 's status at time $t$. We propose the following longitudinal influence model:

$$
\begin{aligned}
y_{i, t+1} & \sim \operatorname{Bernoulli}\left(p_{i, t+1}\right), \\
\operatorname{logit}\left(p_{i, t+1}\right) & =\gamma+\beta_{0} y_{i, t}, \quad i=1,2, \ldots, n \text { and } t=1,2, \ldots, T-1,
\end{aligned}
$$


where $\gamma$ is the intercept and $\beta_{0}$ is the coefficient for the time-lagged status $y_{i, t}$.

If the true degrees of influence is $D^{*}>0$, then each individual may be influenced by anyone to whom the individual is connected by a path with length no more than $D^{*}$. So each ego $i$ 's binary status at time $t+1$ depends on ego $i$ 's status at time $t$ and the status of ego $i$ 's alters with degrees 1 to $D^{*}$ at time $t$. We propose the following longitudinal influence model:

$$
\begin{aligned}
y_{i, t+1} & \sim \operatorname{Bernoulli}\left(p_{i, t+1}\right) \\
\operatorname{logit}\left(p_{i, t+1}\right) & =\gamma+\beta_{0} y_{i, t}+\sum_{d=1}^{D^{*}} \beta_{d} x_{i, t}^{d}, \quad i=1,2, \ldots, n \text { and } t=1,2, \ldots, T-1,
\end{aligned}
$$

where $\gamma$ is the intercept, $\beta_{0}$ is the coefficient for the time-lagged status $y_{i, t}$, $x_{i, t}^{d}$ is the $d$ th-degree influence factor defined in 2.1 and $\beta_{d}$ is its coefficient. In the above model with true degrees of influence $D^{*}>0$, since we assume that each individual $i$ can be influenced by individual $i$ 's alters with degrees 1 to $D^{*}$, we are essentially assuming $\beta_{d} \neq 0$ for $d=1,2, \ldots, D^{*}$.

\section{Hypothesis Testing}

In order to determine the degrees of influence in a dynamic network, we propose a sequential hypothesis testing procedure. This procedure is similar to the forward variable selection in linear regression models, where we 
add one new predictor variable to the model at each time, and implement the goodness of fit test to compare it with the model without the new predictor variable (Hocking, 1976, Everitt and Dunn, 2001). We propose to sequentially test the following hypothesis:

$$
H_{0}: \text { DOI }=D-1 \text { v.s. } H_{1}: \text { DOI } \geq D
$$

We start with $D=1$, and if the null hypothesis is rejected, then we test (4.1) again with $D$ increased by 1 to $D=2$. The procedure continues until the null hypothesis cannot be rejected for a certain value of $D$, and we then report $D-1$ as the degrees of influence.

For the test in (4.1), the null model $M_{0}$ under $H_{0}$ is:

$$
\begin{aligned}
y_{i, t+1} & \sim \operatorname{Bernoulli}\left(p_{i, t+1}\right) \\
\operatorname{logit}\left(p_{i, t+1}\right) & =\gamma+\beta_{0} y_{i, t}+\sum_{d=1}^{D-1} \beta_{d} x_{i, t}^{d}, \quad i=1,2, \ldots, n \text { and } t=1,2, \ldots, T-1 .
\end{aligned}
$$

If the alternative hypothesis is true, based on the discussion after (3.3), the coefficient for the $D$ th-degree influence factor is nonzero. Hence hypothesis (4.1) is testing:

$$
H_{0}: \beta_{D}=0 \text { v.s. } H_{1}: \beta_{D} \neq 0 \text {. }
$$

Under $H_{1}$, the model closest to the null hypothesis is the following alterna- 
tive candidate model $M_{1}$ with $\mathrm{DOI}=D$ :

$y_{i, t+1} \sim \operatorname{Bernoulli}\left(p_{i, t+1}\right)$,

$\operatorname{logit}\left(p_{i, t+1}\right)=\gamma+\beta_{0} y_{i, t}+\sum_{d=1}^{D} \beta_{d} x_{i, t}^{d}, \quad i=1,2, \ldots, n$ and $t=1,2, \ldots, T-1$.

The null model $\left(\beta_{D}=0\right)$ is nested within the alternative candidate model.

In order to implement the test to compare the two models, we need to estimate the parameter $\beta_{D}$ and the variance of the estimator in the alternative candidate model $M_{1}$.

To account for multiple observations of the same individual across different time periods, we use generalized estimating equations (GEEs) (Liang and Zeger, 1986) to estimate $\beta_{D}$ and the variance of the estimator. We first establish the notation for parameter estimation. For the $i$ th individual, let $y_{i}=\left(y_{i, 2}, y_{i, 3}, \ldots, y_{i, T}\right)^{T}$ be the vector of the outcome values and $X_{i}=\left(x_{i, 1}, \ldots, x_{i, T-1}\right)^{T}$ be the matrix of the covariate values, where $x_{i, t}=\left(1, y_{i, t}, x_{i, t}^{1}, \ldots, x_{i, t}^{D}\right)^{T}, t=1, \ldots, T-1$. In the alternative candidate model $M_{1}$, we have $\mathrm{E}\left(y_{i, t+1}\right)=p_{i, t+1}$ and $\operatorname{logit}\left(p_{i, t+1}\right)=x_{i, t}^{T} \beta$, where $\beta=\left(\gamma, \beta_{0}, \beta_{1}, \ldots, \beta_{D}\right)^{T}$. When using GEEs for parameter estimation, we assume an independence working correlation structure. Under certain conditions, this can yield a consistent estimator $\hat{\beta}_{D}$ for $\beta_{D}$ and the variance of the estimator $\hat{\beta}_{D}$ can be consistently estimated by a sandwich estimator 


\subsection{Toy example11}

$\hat{\Sigma}\left(\hat{\beta}_{D}\right)$ Liang and Zeger, 1986). We used the R package geepack (Halekoh, Højsgaard, and Yan, 2006) to solve the GEEs by providing the outcome values $y_{i}$ 's and the matrix of the covariate values $X_{i}$ 's. More details regarding the use of GEEs can be found in Liang and Zeger (1986) and Halekoh, Højsgaard, and Yan (2006).

We use the Wald test with test statistic

$$
W=\frac{\hat{\beta}_{D}^{2}}{\hat{\Sigma}\left(\hat{\beta}_{D}\right)} .
$$

Under the null hypothesis and certain conditions, the test statistic $W$ approximately follows $\chi^{2}(1)$ distribution. For a given significance level $\alpha$, the critical value for the test is $c^{*}=\chi_{1-\alpha}^{2}(1)$, and we reject $H_{0}$ if $W>c^{*}$. For the rest of the paper, we choose $\alpha=0.05$ for all simulation and real data analysis.

\subsection{Toy example}

We use the toy example in Figure 1 to illustrate the sequential testing procedure. The dynamic network in Figure 1 was generated in the follow way. At time $t=1$, we generated a network from the Erdős-Rényi model $\operatorname{ER}\left(n, p_{e}\right)$ (Erdős and Rényi, 1960), where $n$ is the number of nodes and $p_{e}$ is the edge probability. We set $n=10$ and $p_{e}=0.2$. At the following time step $t, 2 \leq t \leq 5$, the network structure is allowed to change. In 
particular, we assume that for every pair of $(i, j), a_{i j, t}$ equals to $a_{i j, t-1}$ with probability 0.95 , and equals to $1-a_{i j, t-1}$ with probability 0.05 . We assigned the smoking status for each node at time $t=1$ based on $\operatorname{Bernoulli}\left(p_{m}\right)$ with $p_{m}=0.2$. At time $t, 2 \leq t \leq 5$, each node's status was generated according to the longitudinal influence model in $(3.3)$, where we set the degrees of influence $D^{*}=1$, parameters $\gamma=-3, \beta_{0}=4$, and $\beta_{1}=4$. In Figure 1 , gray nodes denote smokers and white nodes denote non-smokers.

To explore the degrees of influence in the toy example, we set the significance level $\alpha=0.05$ and started with testing: $H_{0}:$ DOI $=0$ v.s. $H_{1}:$ DOI $\geq 1$. The $d$ th-degree influence factor $x_{i, t}^{d}$ for each individual can be calculated based on Equation (2.1). To get the estimates of the parameters in Equation $(3.3)$ and the corresponding estimated variance, we solved the GEEs under independence working correlation structure using the $\mathrm{R}$ package geepack (Halekoh, Højsgaard, and Yan, 2006). Given $y_{i, t}$ and $x_{i, t}^{1}$ for $i=1,2, \ldots, 10$ and $t=1,2, \ldots, 5$, we obtained the estimate $\hat{\beta}_{1}=6.191$ and the estimated variance $\hat{\Sigma}\left(\hat{\beta}_{1}\right)=2.401$. The test statistic is:

$$
W=\frac{\hat{\beta}_{1}^{2}}{\hat{\Sigma}\left(\hat{\beta}_{1}\right)}=15.961,
$$

which is larger than the critical value $\chi_{0.95}^{2}(1)=3.841$. The null hypothesis $H_{0}:$ DOI $=0$ is rejected.

Then we tested $H_{0}:$ DOI $=1$ v.s. $H_{1}:$ DOI $\geq 2$. Given $y_{i, t}$ and $x_{i, t}^{d}$ 
for $d=1,2, i=1,2, \ldots, 10$ and $t=1,2, \ldots, 5$, we obtained the estimate $\hat{\beta}_{2}=1.147$ and the estimated variance $\hat{\Sigma}\left(\hat{\beta}_{2}\right)=1.659$. The test statistic is:

$$
W=\frac{\hat{\beta}_{2}^{2}}{\hat{\Sigma}\left(\hat{\beta}_{2}\right)}=0.792
$$

which is smaller than the critical value 3.841. The null hypothesis $H_{0}$ : DOI $=1$ cannot be rejected, and the degrees of influence in the toy example is reported to be 1 based on the sequential test.

\section{Theoretical Properties}

In this section, we provide some theoretical properties of the proposed sequential testing procedure. The following theorem shows how the level and power of the test change as the network size increases.

Theorem 1. Suppose we observe a dynamic network with $n$ nodes at time $1, \ldots, T$ and binary vectors $Y_{1}, \ldots, Y_{n}$ indicating the presence or absence of a trait for each individual in the network. Let $D^{*}$ be the true degrees of influence in the network. Let $W$ in (4.5) be the proposed test statistic for testing $H_{0}: D O I=D-1$ v.s. $H_{1}: D O I \geq D$, with $W$ estimated from the observations that are independent across individuals. Let $\alpha$ be the significance level and $c^{*}$ be the critical value of the test. We have the following results. 
(a) The level of the test $P\left(W>c^{*} \mid D O I=D-1\right) \longrightarrow \alpha$ as $n \rightarrow \infty$ for $D-1=D^{*}$.

(b) The power of the test $P\left(W>c^{*} \mid D O I \geq D\right) \longrightarrow 1$ as $n \rightarrow \infty$ for all $1 \leq D \leq D^{*}$

The proof of Theorem 1 is in Appendix A.1. The above theorem indicates that the level of the test goes to the significance level $\alpha$ and the power of the test goes to 1 as the network size $n \rightarrow \infty$. This shows that the test can always tell the difference between the null and the alternative hypotheses when the network size is large. This also indicates that, for large networks, the true degrees of influence can be detected by our method with high probability.

The theorem requires that the test statistic $W=\hat{\beta}_{D}^{2} / \hat{\Sigma}\left(\hat{\beta}_{D}\right)$ is estimated based on observations that are independent across individuals, so that the theoretical properties of estimates using GEEs are applicable here. To obtain independent data across individuals, a convenient assumption is that $y_{1, t}, \ldots, y_{n, t}$ are independent conditional on all the observations at time $t-1$. Under this assumption, there are different ways to obtain independent data. For example, observations $y_{i, t}$ 's at $t=2$ are conditionally independent given $y_{i, t}$ 's at $t=1$. Furthermore, $y_{i, t}$ 's at $t=2 k(k=1,2, \ldots)$ are conditionally independent given $y_{i, t}$ 's at $t=2 k-1$. Since these independent data do not 
make full use of the information in the observed data, in practice, using all observations $y_{i, t}$, as discussed in Section 4, tends to perform better. Therefore, we use full data in our simulation studies and real data analysis.

In the proof about the power of the test (part (b) of the theorem), the test statistic $W$ is estimated based on the true model in the alternative hypothesis. In practice, the true model is not known in the middle of the sequential test, so we estimate $W$ based on the alternative candidate model $M_{1}$ in (4.4). This approach works well in the simulation studies and real data analysis. This kind of approach has also been suggested in sequential testing for forward variable selection in linear regression models Hocking, 1976; Everitt and Dunn, 2001). In fact, our simulation shows that the test based on $M_{1}$ is even more powerful than the test based on the true model for testing (4.1) with $D<D^{*}$. An intuitive explanation might be because the estimates based on the alternative candidate model $M_{1}$ needs to reflect the additional influence from distances larger than $D$, which makes it easier to reject the null hypothesis of DOI $=D-1$. 


\section{Simulation Results}

\subsection{Detecting the degrees of influence}

In this section, we show the performance of our proposed test procedure in detecting the degrees of influence in dynamic networks. We generated a network at time $t=1$ from the Erdős-Rényi model $\operatorname{ER}\left(n, p_{e}\right)$, where $n$ is the number of nodes and $p_{e}$ is the edge probability. At the following time step $t, 2 \leq t \leq 5$, the network structure changes in the following way. For each pair of $\{i, j\}$, if $a_{i j, t-1}=1$, then $a_{i j, t}=1$ with probability 0.95 and $a_{i j, t}=0$ with probability 0.05 . If $a_{i j, t-1}=0$, then $a_{i j, t}=1$ with probability $p_{\text {change }}=0.05 p_{e} /\left(1-p_{e}\right)$ and $a_{i j, t}=0$ with probability $1-p_{\text {change }}$. At time $t=1$, we assigned the status $y_{i, 1}$ for each individual from Bernoulli $\left(p_{m}\right)$ with $p_{m}=0.2$. For time $t=2,3,4,5$, each individual's status was generated according to the longitudinal influence model in (3.3), where we set the true degrees of influence $D^{*}=2$, parameters $\gamma=-3$ and $\beta_{0}=4$. The values of $\beta_{1}$ and $\beta_{2}$, together with $n$ and $p_{e}$, are presented in Table 1 . For each set of parameter values, we generated data and applied our proposed method to detect the DOI. We ran 50 trials for each simulation and the results are presented in Table 1 .

In Table 1, the first column gives the parameter settings for the network 
6.1 Detecting the degrees of influence17

\begin{tabular}{|c|c|c|c|}
\hline$\left(n, p_{e}, \beta_{1}, \beta_{2}\right)$ & $<D^{*}$ & $=D^{*}$ & $>D^{*}$ \\
\hline$(300,0.02,3,3)$ & 0 & 47 & 3 \\
\hline$(500,0.02,3,3)$ & 0 & 49 & 1 \\
\hline$(1000,0.01,2,2)$ & 4 & 44 & 2 \\
\hline$(3000,0.005,2,2)$ & 1 & 47 & 2 \\
\hline$(5000,0.003,2,2)$ & 0 & 47 & 3 \\
\hline
\end{tabular}

Table 1: Results for detecting the degrees of influence in different parameter settings.

size $n$, the edge probability $p_{e}$ and the coefficients $\beta_{1}, \beta_{2}$ in the model in Equation (3.3). The columns " $<D^{*}, "=D^{*}$, and $">D^{*}$ represent the number of trials the DOI detected using our proposed method is smaller than, equal to, and larger than the true DOI, respectively. From Table 1 , we can see that our proposed test procedure can detect the true DOI in most cases.

We also considered detecting the DOI when the network structure is fixed for the whole time period $1 \leq t \leq 5$. With the same parameter settings as the above simulation, we got similar results as Table 1. This shows that our proposed test procedure works well for both fixed and varying network structures. 
6.2 Levels and powers for the tests18

\subsection{Levels and powers for the tests}

In this section, we show the level and power of our proposed test for different parameter settings and different true degrees of influence. We generated a network at time $t=1$ from the Erdős-Rényi model $\operatorname{ER}\left(n, p_{e}\right)$. At the following time step $t, 2 \leq t \leq 5$, the network structure changes in the following way. For each pair of $\{i, j\}$, if $a_{i j, t-1}=1$, then $a_{i j, t}=1$ with probability 0.95 and $a_{i j, t}=0$ with probability 0.05 . If $a_{i j, t-1}=0$, then $a_{i j, t}=1$ with probability $p_{\text {change }}=0.05 p_{e} /\left(1-p_{e}\right)$ and $a_{i j, t}=0$ with

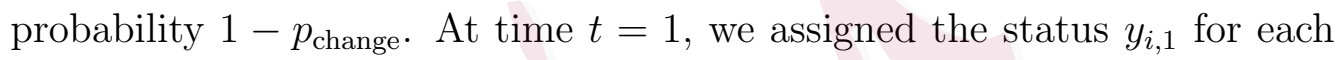
individual from Bernoulli $\left(p_{m}\right)$ with $p_{m}=0.2$. For time $t=2,3,4,5$, each individual's status was generated according to the longitudinal influence model in $(3.3)$. For a given DOI $D^{*}$, we generated data with true DOI $=D^{*}$ and $\beta_{1}, \ldots, \beta_{D^{*}}$ set to pre-specified values. We then estimated the power of the test $H_{0}:$ DOI $=D-1$ v.s. $H_{1}:$ DOI $\geq D$ for $1 \leq D \leq D^{*}$ and the level of the test $H_{0}:$ DOI $=D-1$ v.s. $H_{1}:$ DOI $\geq D$ for $D-1=D^{*}$. In practice, when the size of the network $n$ gets larger, the network may become sparse and the edge probability may decrease. So we assigned smaller values to $p_{e}$ for larger networks. For the rest of this section, we set $\gamma=-3$ and $\beta_{0}=4$ in model (3.3). We ran 100 trials for each simulation setting to obtain the level and power. 


\subsubsection{Testing when true DOI is 0}

In this section, we assume the true DOI $D^{*}=0$, and the data were generated from model $(3.2)$. We consider testing $H_{0}:$ DOI $=0$ v.s. $H_{1}: \quad$ DOI $\geq 1$. Since $H_{0}$ represents the true DOI, we only look at the level of the test. The first column of Table 2 gives the parameter values for the network size $n$, the edge probability $p_{e}$ at time $t=1$. We ran 100 trials for each simulation to estimate the level. From the results in Table2, we can see that our test procedure can achieve the level around the pre-specified $\alpha=0.05$.

\begin{tabular}{|c|c|}
\hline$\left(n, p_{e}\right)$ & Level \\
\hline$(500,0.02)$ & 0.06 \\
\hline$(1000,0.01)$ & 0.04 \\
\hline$(3000,0.005)$ & 0.06 \\
\hline$(5000,0.003)$ & 0.07 \\
\hline
\end{tabular}

Table 2: Levels for testing $H_{0}$ : DOI $=0$ v.s. $H_{1}$ : DOI $\geq 1$ when the true DOI $D^{*}=0$. 


\subsubsection{Testing when true DOI is 1}

In this section, we assume the true DOI $D^{*}=1$. The first column of Table 3 gives the parameter values for the network size $n$, the edge probability $p_{e}$ at time $t=1$ and the coefficient $\beta_{1}$ in model $(3.3)$. The non-zero $\beta_{1}$ is for generating data with true DOI $D^{*}=1$. We first consider testing $H_{0}:$ DOI $=0$ v.s. $H_{1}:$ DOI $\geq 1$ and report the power of the test in the second column of Table 3 . Then we consider testing $H_{0}: \quad$ DOI $=1$ v.s. $H_{1}:$ DOI $\geq 2$ and report the level of the test in the third column of Table 3. We ran 100 trials for each simulation to estimate the level and power.

From the results in Table 3, we can see that our test procedure can achieve the level around the pre-specified $\alpha=0.05$. The power of our test is close or equal to 1 in all settings. This shows that our test is powerful in all of the above parameter settings when the true DOI is 1 .

\subsubsection{Testing when true DOI is 2}

In this section, we assume the true DOI $D^{*}=2$. The first column of Table 4 gives the parameter values for the network size $n$, the edge probability $p_{e}$ at time $t=1$, and the coefficients $\beta_{1}$ and $\beta_{2}$ in model 3.3 . We first consider testing $H_{0}:$ DOI $=0$ v.s. $H_{1}:$ DOI $\geq 1$ and report the power of the test in the second column of Table 4 (denoted by Power-1). Then we 
6.2 Levels and powers for the tests21

\begin{tabular}{|c|c|c|}
\hline$\left(n, p_{e}, \beta_{1}\right)$ & Power & Level \\
\hline$(500,0.02,3)$ & 1 & 0.06 \\
\hline$(1000,0.01,3)$ & 1 & 0.05 \\
\hline$(3000,0.005,3)$ & 1 & 0.03 \\
\hline$(5000,0.003,3)$ & 1 & 0.05 \\
\hline$(500,0.02,2)$ & 0.96 & 0.03 \\
\hline$(1000,0.01,2)$ & 1 & 0.06 \\
\hline$(3000,0.005,2)$ & 1 & 0.04 \\
\hline$(5000,0.003,2)$ & 1 & 0.03 \\
\hline
\end{tabular}

Table 3: Powers for testing: $H_{0}$ : DOI $=0$ v.s. $H_{1}$ : DOI $\geq 1$ and levels for testing: $H_{0}$ : DOI $=1$ v.s. $H_{1}:$ DOI $\geq 2$ when the true DOI $D^{*}=1$.

consider testing $H_{0}:$ DOI $=1$ v.s. $H_{1}:$ DOI $\geq 2$ and report the power of the test in the third column of Table 4 (denoted by Power-2). Finally we consider testing $H_{0}:$ DOI $=2$ v.s. $H_{1}:$ DOI $\geq 3$ and report the level of the test in the fourth column of Table 4. We ran 100 trials for each simulation to estimate the level and power.

In Table 4, the coefficients $\beta_{1}$ and $\beta_{2}$ were chosen to be the same. In some situations, the influence from the second-degree alters may be weaker than the influence from the first-degree alters, so we set $\beta_{2}<\beta_{1}$ in Table 5 
6.2 Levels and powers for the tests22

\begin{tabular}{|c|c|c|c|}
\hline$\left(n, p_{e}, \beta_{1}, \beta_{2}\right)$ & Power-1 & Power-2 & Level \\
\hline$(500,0.02,3,3)$ & 1 & 0.94 & 0.03 \\
\hline$(1000,0.01,3,3)$ & 1 & 1 & 0.06 \\
\hline$(3000,0.005,3,3)$ & 1 & 1 & 0.02 \\
\hline$(5000,0.003,3,3)$ & 1 & 1 & 0.07 \\
\hline$(500,0.02,2,2)$ & 1 & 0.55 & 0.05 \\
\hline$(1000,0.01,2,2)$ & 1 & 0.91 & 0.03 \\
\hline$(3000,0.005,2,2)$ & 1 & 1 & 0.04 \\
\hline$(5000,0.003,2,2)$ & 1 & 1 & 0.06 \\
\hline
\end{tabular}

Table 4: Powers for testing: $H_{0}$ : DOI $=0$ v.s. $H_{1}$ : DOI $\geq 1$ and $H_{0}:$ DOI $=1$ v.s. $H_{1}:$ DOI $\geq 2$, and levels for testing: $H_{0}:$ DOI $=2$ v.s. $H_{1}:$ DOI $\geq 3$ when the true DOI $D^{*}=2$ and $\beta_{2}=\beta_{1}$.

and re-ran the same tests. The results are presented in Table 5.

From Tables 4 and 5, we can see that our proposed method can still preserve the level of the test with type I error close to the pre-specified level $\alpha=0.05$. The power of the test $H_{0}$ : DOI $=0$ v.s. $H_{1}$ : DOI $\geq 1$ is always 1 . The power of the test $H_{0}:$ DOI $=1$ v.s. $H_{1}:$ DOI $\geq 2$ increases to about 1 as the network size increases. For fixed network size $n$ and edge probability $p_{e}$, the test $H_{0}: \mathrm{DOI}=1$ v.s. $H_{1}:$ DOI $\geq 2$ is more powerful for 
6.2 Levels and powers for the tests23

\begin{tabular}{|c|c|c|c|}
\hline$\left(n, p_{e}, \beta_{1}, \beta_{2}\right)$ & Power-1 & Power-2 & Level \\
\hline$(500,0.02,3,2.25)$ & 1 & 0.93 & 0.04 \\
\hline$(1000,0.01,3,2.25)$ & 1 & 1 & 0.06 \\
\hline$(3000,0.005,3,2.25)$ & 1 & 1 & 0.04 \\
\hline$(5000,0.003,3,2.25)$ & 1 & 1 & 0.07 \\
\hline$(500,0.02,2,1.5)$ & 1 & 0.30 & 0.05 \\
\hline$(1000,0.01,2,1.5)$ & 1 & 0.49 & 0.04 \\
\hline$(3000,0.005,2,1.5)$ & 1 & 0.84 & 0.03 \\
\hline$(5000,0.003,2,1.5)$ & 1 & 0.93 & 0.05 \\
\hline
\end{tabular}

Table 5: Powers for testing: $H_{0}$ : DOI $=0$ v.s. $H_{1}$ : DOI $\geq 1$ and $H_{0}$ : DOI $=1$ v.s. $H_{1}:$ DOI $\geq 2$, and levels for testing: $H_{0}$ : DOI $=2$ v.s. $H_{1}:$ DOI $\geq 3$ when the true DOI $D^{*}=2$ and $\beta_{2}<\beta_{1}$.

larger values of $\beta_{2}$. This is not surprising since larger values of $\beta_{2}$ indicate stronger influence from second-degree alters, which makes it easier to detect the mis-specified null hypothesis of no influence from second-degree alters. For fixed values of $\beta_{1}$ and $\beta_{2}$, the power of the test increases as the network size increases. 
6.2 Levels and powers for the tests24

\subsubsection{Testing when true DOI is 3}

In this section, we assume the true DOI $D^{*}=3$. The first column of Table 6 gives the parameter values for the network size $n$, the edge probability $p_{e}$ at time $t=1$, and the coefficients $\beta_{1}, \beta_{2}$ and $\beta_{3}$ in model (3.3). We consider testing $H_{0}:$ DOI $=D-1$ v.s. $H_{1}:$ DOI $\geq D$ for $D=1,2,3$, and report the power of the test in the second, third and fourth columns of Table 6, respectively (denoted by Power-1, Power-2, Power-3). Then we consider testing $H_{0}:$ DOI $=3$ v.s. $H_{1}:$ DOI $\geq 4$ and report the level of the test in the fifth column of Table 6. We ran 100 trials for each simulation to estimate the level and power.

In Table 6, the coefficients $\beta_{1}=\beta_{2}=\beta_{3}$ were chosen to be the same. In some situations, the influence from the $D$ th-degree alters may be weaker than the influence from the $(D-1)$ th-degree alters, so we set $\beta_{3}<\beta_{2}<\beta_{1}$ in Table 7 and re-ran the same tests. The results are presented in Table 7.

From Tables 6 and 7, we can see that the correct level is achieved in different settings. The powers of the tests $H_{0}$ : DOI $=0$ v.s. $H_{1}$ : DOI $\geq 1$ and $H_{0}:$ DOI $=1$ v.s. $H_{1}:$ DOI $\geq 2$ are always around 1 . With the same parameter settings for $\beta_{i}$ 's $(i=1,2,3)$, the power of the test $H_{0}$ : $\mathrm{DOI}=$ 2 v.s. $H_{1}$ : DOI $\geq 3$ increases as the network size gets larger. For fixed network size $n$ and edge probability $p_{e}$, the test $H_{0}$ : DOI $=2$ v.s. $H_{1}:$ DOI 
6.2 Levels and powers for the tests25

\begin{tabular}{|c|c|c|c|c|}
\hline$\left(n, p_{e}, \beta_{1}, \beta_{2}, \beta_{3}\right)$ & Power-1 & Power-2 & Power-3 & Level \\
\hline$(500,0.02,3,3,3)$ & 1 & 1 & 0.47 & 0.05 \\
\hline$(1000,0.01,3,3,3)$ & 1 & 1 & 0.69 & 0.03 \\
\hline$(3000,0.005,3,3,3)$ & 1 & 1 & 0.82 & 0.07 \\
\hline$(5000,0.003,3,3,3)$ & 1 & 1 & 0.95 & 0.06 \\
\hline$(500,0.02,2,2,2)$ & 1 & 1 & 0.35 & 0.04 \\
\hline$(1000,0.01,2,2,2)$ & 1 & 1 & 0.68 & 0.03 \\
\hline$(3000,0.005,2,2,2)$ & 1 & 1 & 0.78 & 0.06 \\
\hline$(5000,0.003,2,2,2)$ & 1 & 1 & 0.90 & 0.05 \\
\hline
\end{tabular}

Table 6: $\quad$ Powers for testing: $H_{0}:$ DOI $=D-1$ v.s. $H_{1}$ : DOI $\geq D$ for $D=1,2,3$, and levels for testing: $H_{0}$ : DOI $=3$ v.s. $H_{1}:$ DOI $\geq 4$ when the true DOI $D^{*}=3$ and $\beta_{3}=\beta_{2}=\beta_{1}$.

$\geq 3$ is more powerful for larger values of $\beta_{3}$, which is consistent with our intuition.

We also re-ran all simulations regarding levels and powers in Section 6.2 with fixed network structure for the whole time period $1 \leq t \leq 5$. With the same parameter settings as the above simulations, we got similar results as Tables 2 to 7 . This shows that our proposed test procedure also works well for dynamic networks with fixed network structure. 


\begin{tabular}{|c|c|c|c|c|}
\hline$\left(n, p_{e}, \beta_{1}, \beta_{2}, \beta_{3}\right)$ & Power-1 & Power-2 & Power-3 & Level \\
\hline$(3000,0.005,3,2.25,1.5)$ & 1 & 1 & 0.44 & 0.06 \\
\hline$(5000,0.003,3,2.25,1.5)$ & 1 & 1 & 0.61 & 0.05 \\
\hline$(10000,0.002,3,2.25,1.5)$ & 1 & 1 & 0.70 & 0.05 \\
\hline$(3000,0.005,2,1.5,1)$ & 1 & 0.99 & 0.24 & 0.03 \\
\hline$(5000,0.003,2,1.5,1)$ & 1 & 1 & 0.35 & 0.07 \\
\hline$(10000,0.002,2,1.5,1)$ & 1 & 1 & 0.47 & 0.03 \\
\hline
\end{tabular}

Table 7: $\quad$ Powers for testing: $H_{0}:$ DOI $=D-1$ v.s. $H_{1}:$ DOI $\geq D$ for $D=1,2,3$, and levels for testing: $H_{0}:$ DOI $=3$ v.s. $H_{1}:$ DOI $\geq 4$ when the true DOI $D^{*}=3$ and $\beta_{3}<\beta_{2}<\beta_{1}$.

\section{Real Data Analysis}

\section{$7.1 \quad$ Higgs Twitter data}

Twitter is a popular American social networking site which has a microblogging system that allows users to post and interact with posts called "tweets". In this section, we analyze the Higgs Twitter Dataset collected by De Domenico et al. (2013) and is available at https://snap.stanford.edu/data/higgstwitter.html. This data was built by keeping track of the spreading process on Twitter before, during and after the announcement on July 4, 2012 of 
the discovery of a new particle with the elusive Higgs boson features.

The dataset contains a network of Twitter users who have posted messages on Twitter about this discovery between July 1, 2012 and July 7, 2012. Nodes in the network correspond to users and an edge from node $i$ to $j$ means node $i$ follows node $j$. The dataset also contains interactions (including retweet, mention and reply) between users with time stamp. Here, we focus on the mention behavior as a feature of interest, which indicates a user mentioned other users when he/she posted some tweets on Twitter related to the Higgs boson discovery. This feature is represented by a binary random variable $y_{i, t}$, where $y_{i, t}=1$ indicates that user $i$ already mentioned other users in the tweets about the discovery before a certain time $t$ and $y_{i, t}=0$ otherwise.

Since the network in the original dataset has a large number of nodes, we consider a subset of the network by choosing the node that first showed the interested feature, and then selecting the nodes that have a path with length no more than 2 to this node. This subnetwork has 1757 nodes and is shown in Figure 2, The network structure does not change in this data.

Since the announcement of the discovery was on July 4, 2012, the spread of the feature of interest after the announcement might not come from social influence in the network. Therefore the time interval of interest is from July 


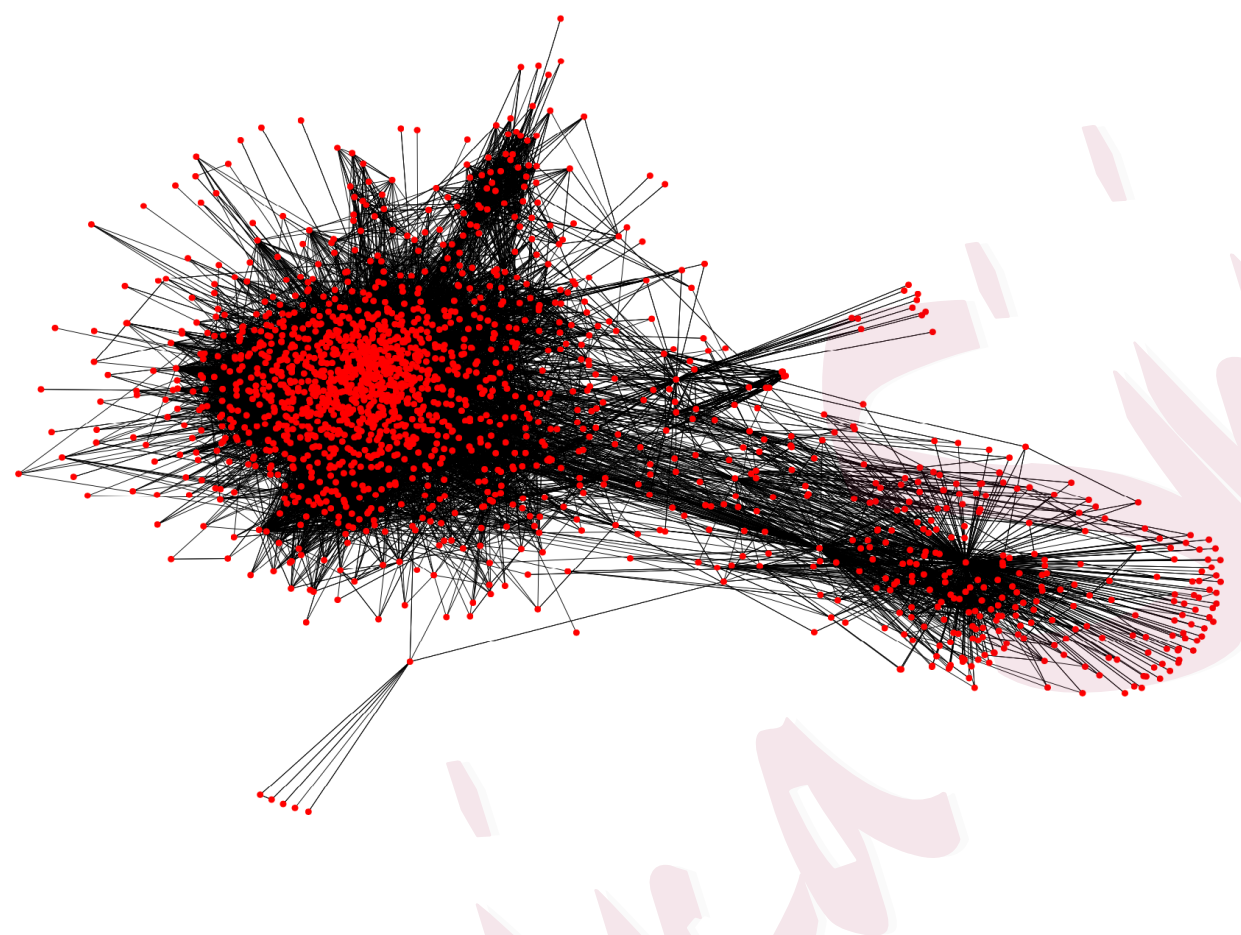

Figure 2: Higgs Twitter network.

1, 2012 to July 3, 2012. We divided this interval into six time steps with $t_{1}$ be $12 \mathrm{pm}$ of $7 / 1 / 2012$ and $t_{6}$ be $12 \mathrm{am}$ of $7 / 4 / 2012$, and the gap between two neighboring time steps is 12 hours.

We applied our sequential hypothesis test to determine the degrees of influence for the mention behavior. We started with testing $H_{0}:$ DOI $=0$ v.s. $H_{1}:$ DOI $\geq 1$, and the test statistic $W$ in 4.5 is 9.43 , which is larger than the critical value $\chi_{0.95}^{2}(1)=3.841$. So we rejected the null hypothesis and continued to test $H_{0}:$ DOI $=1$ v.s. $H_{1}:$ DOI $\geq 2$. This time 
$W=57.76$ which is still larger than the critical value, so the null hypothesis is again rejected. Then we tested: $H_{0}:$ DOI $=2$ v.s. $H_{1}:$ DOI $\geq 3$, and the test statistic $W=0.0019$ is smaller than the critical value. So the null hypothesis $H_{0}$ cannot be rejected and we report the degrees of influence is 2. That means the mention behavior in Twitter can be influenced by an individual's followees as well as his/her followees' followees. Note that there are many length-3 paths in the subnetwork, and every node in the subnetwork has some third-degree alters. So accepting $H_{0}:$ DOI $=2$ is not because there are not enough length-3 paths.

\subsection{Digg data}

Digg is a social news website with a curated front page, which selects interesting stories related to viral Internet issues, science, and political news for Internet audience. Users can read and share the most popular and interesting stories on the internet. Digg2009 Dataset, collected by Hogg and Lerman (2012), contains data of the stories promoted to Digg's front page based on user's votes in one month in 2009. The data is available at https://www.isi.edu/ Terman/downloads/digg2009.html. This dataset is anonymized and has the voting records for 3553 different stories during that month. The voting record for each story contains the ID of the voter and 
the time stamp of the vote. In addition, the dataset contains links of the voters and the time stamp of the formation of the link. Here a link from node $i$ to $j$ means user $i$ is a fan/follower of user $j$.

We consider users' votes for the most voted story (which is story 714) as the feature of interest. This feature is represented by a binary random variable $y_{i, t}$, where $y_{i, t}=1$ means user $i$ voted for story 714 before time $t$ and $y_{i, t}=0$ otherwise. Since the original network has a large number of nodes, we consider a subset of the network by choosing the node that made the first vote for story 714 , and then selecting the nodes directly following this node at the time when the first vote for story 714 was made. This subnetwork has 1408 nodes.

For the selected subnetwork, a total of 304 votes were made for story 714. Right after the first vote at 17:42:46 on June 25, 2009, there were 169 votes before 21:00:00 of the same day. This fast increase of votes probably was not due to social influence. Also there were only 12 votes after June 26, 2009, and we believe social influence was very weak by that time. So the time interval of interest is from 21:00:00 of June 25, $2009\left(t_{1}\right)$ to 00:00:00 of June 27, $2009\left(t_{10}\right)$, during which the number of votes increased from 169 to 292. We divided this interval into ten time steps and the gap between two neighboring time steps is 3 hours. The network structure also changed 
slightly during the selected time interval. The subnetwork at time $t_{1}$ is shown in Figure 3 .

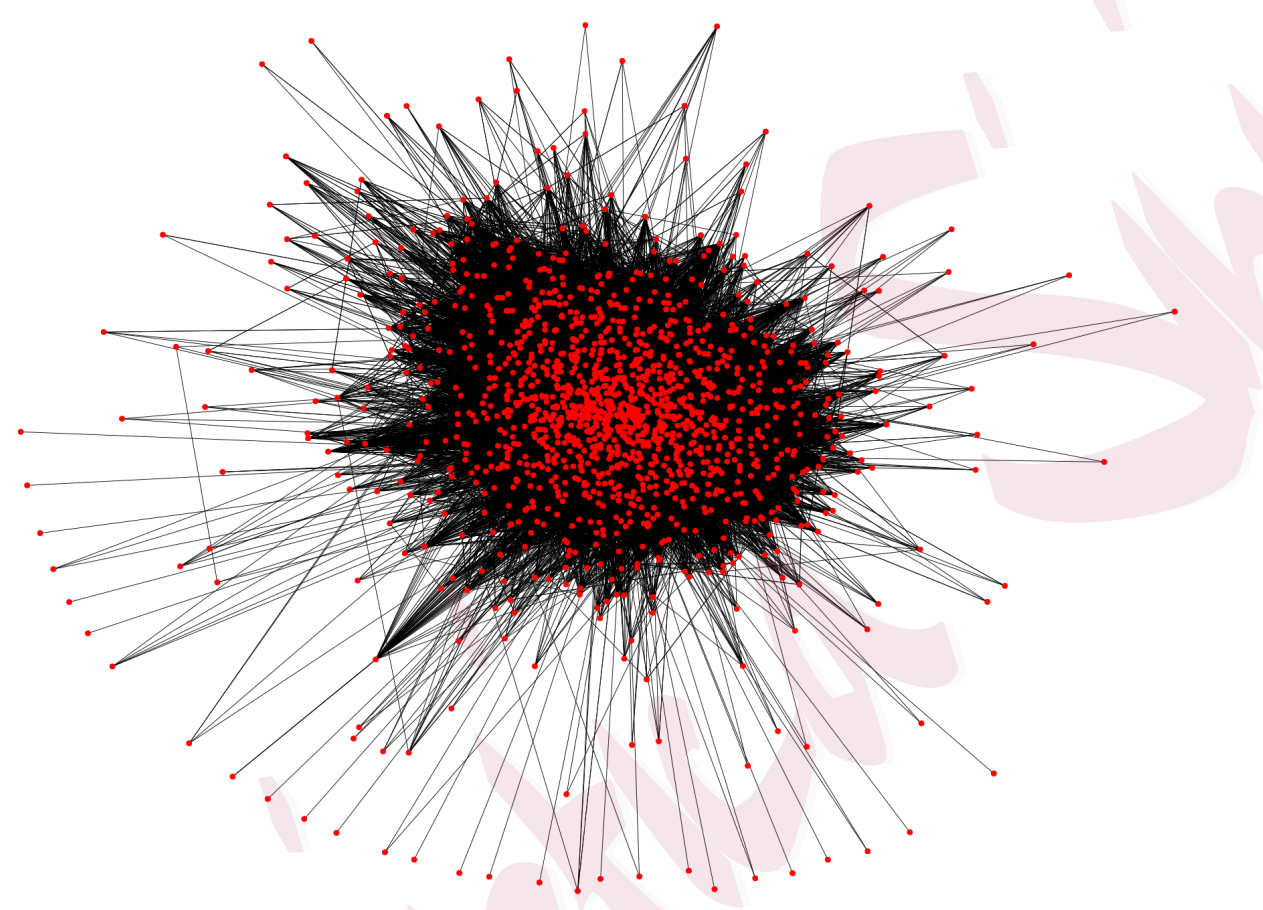

Figure 3: Digg network at time $t_{1}$.

We applied our sequential hypothesis test to determine the degrees of influence for the voting behavior for story 714 . We tested $H_{0}:$ DOI $=D-1$ v.s. $H_{1}$ : DOI $\geq D$ for $D=1,2$ and 3 , and the test statistic $W$ is 4.02 , 28.80 and 56.40, respectively, which are all larger than the critical value $\chi_{0.95}^{2}(1)=3.841$. So the null hypothesis $H_{0}$ for all three tests are rejected. Then we continued to test: $H_{0}:$ DOI $=3$ v.s. $H_{1}:$ DOI $\geq 4$, and the test 
statistic $W=0.87$ is smaller than the critical value. So null hypothesis $H_{0}$ cannot be rejected and we report the degrees of influence is 3. This shows that users' voting behavior in Digg network can be influenced by their directly connected neighbors, their neighbor's neighbors and their thirddegree alters. Note that there are many length-4 paths in the subnetwork. On average, each individual has more than 50 fourth-degree alters that can potentially influence this individual. So the fact that we cannot reject $H_{0}:$ DOI $=3$ is not because there are not enough length-4 paths.

\section{Discussion}

In this paper, we build a longitudinal influence model for dynamic networks with various degrees of influence. We propose a sequential testing procedure to determine the degrees of influence in dynamic networks. We also provide theoretical justification for our proposed test and show that the power of the test goes to one as the network size goes to infinity. Our proposed test performs well in simulation studies and real data analysis. The sequential testing procedure may involve multiple tests, but since the degrees of influence is usually small (often no more than three), we only need to perform the test a few times in most cases. Therefore we do not consider the issue with multiple tests in this paper. 
The proposed longitudinal model and sequential test for dynamic networks are very different from the testing procedure for static networks (Christakis and Fowler, 2013, Su, 2019). It would be of interest to consider

extensions of the method proposed by $\mathrm{Su}(2019)$ to dynamic networks, and compare the performance with the approach in this paper. A related topic is to predict how a certain opinion/behavior spread in a network, and how individuals' behavior will change in the future based on social influence. Missing values in individuals' status or missing edges between individuals is quite common in real data. Developing methods to deal with missing data is also very useful in practice. Another interesting problem is to test whether the social influence decreases as the social distance increases. This is beyond the scope of this paper and will be studied in our future work.

\section{Acknowledgements}

This work was supported in part by National Science Foundation grants CCF-1934986 and DMS-2015561. We thank the Editor, associate editor, and two referees for their constructive comments. 


\section{Appendix A}

\section{A.1 Proof of Thereom 1}

\section{Part (a):}

For testing: $H_{0}:$ DOI $=D-1$ v.s. $H_{1}:$ DOI $\geq D$, the test statistic is $\hat{\beta}_{D}^{2} / \hat{\Sigma}\left(\hat{\beta}_{D}\right)$ in 4.5 , where $\hat{\beta}_{D}$ is the estimate based on the generalized estimating equations and $\hat{\Sigma}\left(\hat{\beta}_{D}\right)$ is the sandwich estimator for the variance of $\hat{\beta}_{D}$. Under $H_{0}$, the longitudinal influence model is:

$$
\begin{aligned}
y_{i, t+1} & \sim \operatorname{Bernoulli}\left(p_{i, t+1}\right) \\
\operatorname{logit}\left(p_{i, t+1}\right) & =\gamma+\beta_{0} y_{i, t}+\sum_{d=1}^{D} \beta_{d} x_{i, t}^{d}
\end{aligned}
$$

where $\beta_{D}=0$.

By the property of generalized estimating equations (Liang and Zeger, 1986), $\hat{\beta}_{D}$ is a consistent estimator for $\beta_{D}$ and

$$
\sqrt{n} \hat{\beta}_{D} \stackrel{d}{\longrightarrow} N\left(0, V(\beta)_{D+2, D+2}\right)
$$

where $V(\beta)_{D+2, D+2}=\lim _{n \rightarrow \infty} n V_{n}(\beta)_{D+2, D+2}$ and $V_{n}(\beta)=H_{1}(\beta)^{-1} H_{2}(\beta) H_{1}(\beta)^{-1}$ (Liang and Zeger, 1986). Thus,

$$
\frac{\hat{\beta}_{D}^{2}}{V(\beta)_{D+2, D+2} / n}=\frac{n \hat{\beta}_{D}^{2}}{V(\beta)_{D+2, D+2}} \stackrel{d}{\longrightarrow} \chi^{2}(1) .
$$

Given $n \hat{\Sigma}\left(\hat{\beta}_{D}\right)$ is a consistent estimator for $V(\beta)_{D+2, D+2}$ Liang and Zeger. 
1986) and by Slutsky's theorem, we have

$$
W=\frac{\hat{\beta}_{D}^{2}}{\hat{\Sigma}\left(\hat{\beta}_{D}\right)}=\frac{n \hat{\beta}_{D}^{2}}{n \hat{\Sigma}\left(\hat{\beta}_{D}\right)}=\frac{n \hat{\beta}_{D}^{2}}{V(\beta)_{D+2, D+2}} \frac{V(\beta)_{D+2, D+2}}{n \hat{\Sigma}\left(\hat{\beta}_{D}\right)} \stackrel{d}{\longrightarrow} \chi^{2}(1) .
$$

Since $c^{*}=\chi_{1-\alpha}^{2}(1)$, we have:

$$
P\left(W>c^{*} \mid \mathrm{DOI}=D-1\right) \longrightarrow \alpha .
$$

\section{Part (b):}

Under $H_{1}$, the longitudinal influence model is:

$$
\begin{aligned}
y_{i, t+1} & \sim \operatorname{Bernoulli}\left(p_{i, t+1}\right) \\
\operatorname{logit}\left(p_{i, t+1}\right) & =\gamma+\beta_{0} y_{i, t}+\sum_{d=1}^{D^{*}} \beta_{d} x_{i, t}^{d}
\end{aligned}
$$

where $\beta_{D} \neq 0$ for $1 \leq D \leq D^{*}$.

By the property of generalized estimating equations (Liang and Zeger, 1986), $\hat{\beta}_{D}$ is a consistent estimator for $\beta_{D}$ and

$$
\sqrt{n}\left(\hat{\beta}_{D}-\beta_{D}\right) \stackrel{d}{\longrightarrow} N\left(0, V(\beta)_{D+2, D+2}\right)
$$

where $V(\beta)_{D+2, D+2}=\lim _{n \rightarrow \infty} n V_{n}(\beta)_{D+2, D+2}$ and $V_{n}(\beta)=H_{1}(\beta)^{-1} H_{2}(\beta) H_{1}(\beta)^{-1}$

Liang and Zeger, 1986). Also $n \hat{\Sigma}\left(\hat{\beta}_{D}\right)$ is a consistent estimator for $V(\beta)_{D+2, D+2}$

(Liang and Zeger, 1986).

Since $\hat{\beta}_{D}$ is a consistent estimator for $\beta_{D}$, we have $\hat{\beta}_{D} \stackrel{p}{\longrightarrow} \beta_{D}$. So $\hat{\beta}_{D}^{2} \stackrel{p}{\longrightarrow} \beta_{D}^{2}>0$. Note that $V(\beta)$ is a covariance matrix, so $V(\beta)_{D+2, D+2}$ 
is a finite positive number. Since $n \hat{\Sigma}\left(\hat{\beta}_{D}\right)$ is a consistent estimator of $V(\beta)_{D+2, D+2}$, we have

$$
n \hat{\Sigma}\left(\hat{\beta}_{D}\right) \stackrel{p}{\longrightarrow} V(\beta)_{D+2, D+2},
$$

so $\hat{\Sigma}\left(\hat{\beta}_{D}\right) \stackrel{p}{\longrightarrow} 0$. Since $\hat{\beta}_{D}^{2} \stackrel{p}{\longrightarrow} \beta_{D}^{2}>0$, we have $W=\frac{\hat{\beta}_{D}^{2}}{\hat{\Sigma}\left(\hat{\beta}_{D}\right)} \stackrel{p}{\longrightarrow} \infty$. This shows $P\left(W>c^{*} \mid \mathrm{DOI} \geq D\right) \stackrel{p}{\longrightarrow} 1$, where $c^{*}=\chi_{1-\alpha}^{2}(1)$.

\section{References}

Brakefield, T. A., Mednick, S. C., Wilson, H. W., De Neve, J.-E., Christakis, N. A., and Fowler, J. H. (2014). Same-sex sexual attraction does not spread in adolescent social networks. Archives of Sexual Behavior 43(2), 335-344.

Centola, D. (2010). The spread of behavior in an online social network experiment. Science 329(5996), 1194-1197.

Christakis, N. A., and Fowler, J. H. (2007). The spread of obesity in a large social network over 32 years. New England Journal of Medicine 357(4), 370-379.

Christakis, N. A., and Fowler, J. H. (2008). The collective dynamics of smoking in a large social network. New England Journal of Medicine 358(21), 2249-2258.

Christakis, N. A., and Fowler, J. H. (2013). Social contagion theory: examining dynamic social networks and human behavior. Statistics in Medicine 32(4), 556-577.

De Domenico, M., Lima, A., Mougel, P., and Musolesi, M. (2013). The anatomy of a scientific rumor. Scientific Reports 3, 2980. 
Erdős, P., and Rényi, A. (1960). On the evolution of random graphs. Publ. Math. Inst. Hung. Acad. Sci 5(1), 17-60.

Everitt B. S., and Dunn G. (2001). Applied Multivariate Data Analysis, Edward Arnold, London.

Halekoh, U., Højsgaard, S., and Yan, J. (2006). The R package geepack for generalized estimating equations. Journal of Statistical Software 15(2), 1-11.

Hocking, R. R. (1976). The analysis and selection of variables in linear regression. Biometrics 32, $1-49$.

Hogg, T., and Lerman, K. (2012). Social dynamics of digg. EPJ Data Science 1(1), 5.

Kempe, D., Kleinberg, J., and Tardos, É. (2003). Maximizing the spread of influence through a social network. Proceedings of the Ninth ACM SIGKDD International Conference on Knowledge Discovery and Data Mining, 137-146.

Liang, K.-Y., and Zeger, S. L. (1986). Longitudinal data analysis using generalized linear models. Biometrika 73(1), 13-22.

Mednick, S. C., Christakis, N. A., and Fowler, J. H. (2010). The spread of sleep loss influences drug use in adolescent social networks. PLoS One 5(3).

O’Malley, A. J. (2013). The analysis of social network data: an exciting frontier for statisticians. Statistics in Medicine 32(4), 539-555.

Rosenquist, J. N., Murabito, J., Fowler, J. H., and Christakis, N. A. (2010). The spread of alcohol consumption behavior in a large social network. Annals of Internal Medicine 
152(7), 426-433.

$\mathrm{Su}$, X. (2019). Variational approximation for importance sampling and statistical inference on social influence. PhD thesis, University of Illinois at Urbana-Champaign.

Sun, J., and Tang, J. (2011). A Survey of Models and Algorithms for Social Influence Analysis. In Social Network Data Analytics (Edited by C. C. Aggarwal), 177-214. Springer, Boston.

Valente, T. W. (1995). Network Models of the Diffusion of Innovations. Computational and Mathematical Organization Theory 2, 163-164.

VanderWeele, T. J. (2013). Inference for influence over multiple degrees of separation on a social network. Statistics in Medicine 32(4), 591-596.

Department of Statistics, University of Illinois at Urbana-Champaign, Champaign, IL 61820, U.S.A.

E-mail: xiangc5@illinois.edu

Department of Statistics, University of Illinois at Urbana-Champaign, Champaign, IL 61820, U.S.A.

E-mail: yuguo@illinois.edu 